\title{
émulations
}

\section{Quand l'administration fait le couple par le corps}

\section{Les effets du mariage civil en Suisse}

\author{
Anne Lavanchy \\ Émulations - Revue de sciences sociales \\ 2019, n³2, «Aux frontières de la parenté. Un éclairage par les marges ».
}

\section{Article disponible à l'adresse suivante}

https://ojs.uclouvain.be/index.php/emulations/article/view/lavanchy

\section{Pour citer cet article}

Anne Lavanchy, «Quand l'administration fait le couple par le corps. Les effets du mariage civil en Suisse », Émulations, n 32, Mise en ligne le 20 avril 2020.

DOI : 10.14428/emulations.032.06

Distribution électronique : Université catholique de Louvain (Belgique) : ojs.uclouvain.be

(C) Cet article est mis à disposition selon les termes de la Licence Creative Commons Attribution, Pas d'Utilisation Commerciale 4.0 International. http://creativecommons.org/licenses/by-nc/4.0/

Éditeur : Émulations - Revue de sciences sociales / Presses universitaires de Louvain https://ojs.uclouvain.be/index.php/emulations

ISSN électronique : 1784-5734

$\frac{\text { PUL PRESSES }}{\text { UNIVERSITAIRES }}$ 


\title{
Quand l'administration fait le couple par le corps
}

\author{
Les effets du mariage civil en Suisse
}

Anne Lavanchy ${ }^{1}$

\begin{abstract}
[Résumé] Que révèle du lien conjugal l'analyse de la gestion administrative du mariage et de ses effets légaux ? En analysant le travail des officiers d'état civil en Suisse, cet article présente trois constats. II montre d'abord que dans le cadre du dépistage des unions dites de convenance, les couples « mixtes » font l'objet d'un contrôle accru : l'accès que permet le mariage à la naturalisation facilitée met en évidence les effets attendus, mais invisiblisés au quotidien, du lien conjugal. Dans le sillage des nouvelles études de la parenté, il permet dans un second temps de comprendre les substances qui circulent entre les conjoint.e.s - l'amour et les substances sexuelles. Enfin, faire couple ne relève pas simplement de l'ordre de la loi, mais aussi de celui de la nature : le lien conjugal révèle la nature malléable des femmes. Le corps à corps avec des époux étrangers peut changer leur appartenance nationale, qui se voit attribuer une volatilité problématique.
\end{abstract}

Mots-clés : mariage, parenté, naturalisation, anthropologie des institutions, nation.

\section{Doing couple through bodies at the administration: The effects of civil marriage in Switzerland}

[Abstract] What can the analysis of the administrative management of marriage and its legal effects reveal about the conjugal bond? Analysing the work of civil registrars in Switzerland, this article discusses three findings. Firstly, it shows that the screening of so-called "convenience unions" submits "mixed" couples to an increased control: the fact that marriage allows for facilitated naturalization sheds light on the expected, though invisible in the daily life, effects of the conjugal bond. Drawing on new studies of kinship, it secondly allows considering what substances circulate between spouses - love and sexual substances. Doing couple does not follow the mere order of law but also the order of nature: the conjugal bond reveals the malleable nature of women. Their embodied intercourse with foreigners might change their national belongings, making their reliability as citizens problematic.

Keywords: marriage, kinship, naturalisation, anthropology of street-level bureaucracy, nation.

Que révèle du lien conjugal l'analyse de la gestion administrative du mariage et de ses effets légaux ? En explorant cette question à partir du cas de la Suisse, cet article s'intéresse au mariage tel que défini et produit par des pratiques institutionnelles, ainsi que la manière dont elles éclairent ce lien de parenté spécifique réputé purement social. Selon le Code civil, le mariage est un événement non naturel ${ }^{2}$ : la demande de mariage

\footnotetext{
${ }^{1}$ Haute école de travail social, HES-SO, Genève, Suisse.

2 Voir la note 6 de la directive de l'office fédéral sur la saisie des personnes étrangères dans le registre de l'état civil. En ligne, consulté le 23 mars 2019, URL : https://www.bj.admin.ch/content/ dam/data/bj/gesellschaft/zivilstand/weisungen/weisungen-07/10-08-10-01-f.pdf.
} 
fait l'objet d'une évaluation de la part des officiers, à l'issue de laquelle le mariage peut être ou non célébré. Son traitement contraste en ceci avec celui des naissances et des décès, événements définis comme naturels, dont l'inscription dans les registres est inconditionnelle. Dans sa dimension légale et administrative, le mariage comporte deux caractéristiques conjointes : la première est que les officiers évaluent la légitimité de toute demande de mariage en exerçant leur marge d'appréciation, dans laquelle sont mobilisés affects, empathie et attentes normatives (Lavanchy, 2014). La seconde est qu'il est nécessaire d'en surveiller l'accès, puisque l'établissement d'un lien conjugal administratif a des conséquences légales dans plusieurs domaines, en particulier, comme on le verra, celui de l'appartenance nationale.

En analysant la manière dont la production du lien conjugal ouvre l'accès à certaines ressources étatiques dont sont exclues les personnes qui ne peuvent se marier, l'article est centré sur la seconde de ces caractéristiques. Il s'intéresse notamment à ce que révèle du lien conjugal la possibilité d'accéder à la procédure de naturalisation qualifiée de « facilitée ». La " facilitation » concerne tant la procédure elle-même, rassemblée dans les mains des autorités fédérales (un cas de figure peu courant en Suisse, où les prérogatives communales et cantonales sont prépondérantes) que les conditions formelles et informelles - durée de séjour et intégration³. Le fait qu’il permette d'intégrer à la famille nationale une personne non suisse met en évidence le continuum symbolique, administratif et légal entre la famille et la nation (Fassin, 2008; Hill Collins, 2001) qui constitue le point de départ de ma réflexion.

Le mariage est une forme institutionnelle permettant de créer un couple qui, si elle n'est pas exclusive ni exhaustive, reste singulièrement importante en Suisse ${ }^{4}$ alors qu'elle a diminué dans d'autres pays européens ${ }^{5}$. Son succès relatif s'explique par plusieurs facteurs contextuels, comme le manque de protection du lien de paternité pour les naissances hors mariage et le fait qu'il soit la seule forme légale de protection des conjoint·e·s hétérosexuel-le·s en cas de décès ou de maladie - le partenariat enregistré, en vigueur depuis le $1^{\mathrm{er}}$ janvier 2008, étant réservé aux couples de même sexe. Par ailleurs, le mariage en Suisse permet le regroupement familial entre conjoint.e.s, ce qui explique probablement que les couples mariés formés par une personne suisse et une personne étrangère soient en augmentation proportionnelle, alors que le taux de nuptialité entre personnes suisses tend à reculer ${ }^{6}$.

\footnotetext{
${ }^{3}$ Administration fédérale, 2019. En ligne, consulté le 12 décembre 2019, URL : https://www.eda. admin.ch/countries/france/fr/home/services/nationalite/erleichterte-einbuergerung-ehepartner. html.

${ }^{4}$ Office Fédéral de la Statistique, 2019. En ligne, consulté le 26 mars 2019, URL : https://www.bfs. admin.ch/bfs/fr/home/statistiques/population/mariages-partenaires-divorces/nuptialite.html. ${ }^{5}$ Données Eurostat, compilation sur demande par l'OFS, voir aussi Eurostat 2019. En ligne, consulté le 26 mars 2019, URL : https://ec.europa.eu/eurostat/statistics-explained/index.php/Marriage_ and_divorce_statistics.

${ }^{6}$ Administration fédérale, 2019. En ligne, consulté le 26 mars 2019, URL : https://www.bfs.admin. ch/bfs/fr/home/statistiques/population/mariages-partenaires-divorces/nuptialite.html.
} 
Dénué des atours attractifs de la nouveauté sociale, technologique ou juridique, le mariage suscite peu d'intérêt du côté de la recherche anthropologique. Le regain des études de la parenté se concentre sur les nouvelles formes de liens, en particulier en questionnant la dimension biologisée de la filiation, que ce soit dans le cadre des recompositions familiales (Weber, 2013), ou, plus fréquemment, en rapport avec l'évolution des technologies reproductives, notamment en Suisse, où les recherches portent majoritairement sur les effets sociaux et légaux de la procréation médicale assistée pour les couples hétérosexuels infertiles (Bühler, 2015; Engeli, 2010) et pour les couples homosexuels (Boillet, de Luze, 2018 ; Roca i Escoda, 2013). Le mariage relève du non-vu, de l'implicite et de l'allant de soi ; cependant, il quadrille l'espace social en générant des marges stigmatisantes où sont relégués « tous ceux qui donnent [...] le sentiment de ne pas ratifier l'ordre social du couple [...] et qui apparaissent [...] comme un ferment de contestation sociale et de désordre "écologique” » (Tin, 2003 : s. p.). L’intérêt d'analyser les mariages « qui dérangent » (Porqueres i Gené, 2001) est que ce détour par les marges met en lumière les effets attendus, mais invisiblisés au quotidien, du lien conjugal.

S'inscrivant dans le champ des recherches sur les institutions formelles (Aeby, Berthod, 2011), mon propos s'inspire des nouvelles études de la parenté, qui mettent l'accent sur la dimension processuelle de l'apparentement (relatedness) (Carsten, 2011 ; Strong, Franklin, McKinnon, 2001). Il postule que l'analyse du mariage en tant que catégorie administrative et légale constitue un puissant révélateur de ce que sont les liens conjugaux. En effet, au-delà d'une simple traduction ou application des lois et codes, les activités administratives qui produisent le couple marié ont une vie sociale (Von Benda-Beckmann, Von Benda-Beckmann, Eckert, 2009), qui comprend les actions, paroles et représentations du personnel de guichet (Weller, 2013), la mobilisation de leur « feeling » (Hertz, Martin, Valli, 2004) et les formes de l'exercice du pouvoir discrétionnaire (Laurens, 2008). La compréhension de cet apparentement spécifique implique d'aborder les interrogations suivantes : comment est produit le « couple » marié à partir des identités discrètes que sont les fiancée.e-s ? En quoi le mariage suppose-t-il que quelque chose circule entre les corps ? Qu'est-ce qui rend cette circulation efficace et permet à la parenté de déployer des effets sociaux, légaux et administratifs ? Que disent du lien conjugal les conséquences légales d'une séparation?

Pour répondre à ces questions, l'article fait dialoguer deux ensembles de données empiriques, produites par un travail ethnographique combinant entretiens formels et informels, observation et observation participante7. Ci-après sont mentionnés les noms de Camille, Claude, Dominique et Stéphane. Il s'agit des pseudonymes épicènes d'officiers d'état civil interrogés dans le cadre d'une recherche sur la mise en applica-

\footnotetext{
${ }^{7}$ Les possibilités de participer aux tâches quotidiennes ont été très variables selon les offices et les cantons. J'ai pu observer dans tous les cas les pratiques professionnelles quotidiennes, et dans deux cantons j'ai eu aussi l'occasion d'apprendre à entrer des données dans le système informatique, d'effectuer la saisie de ces données pour les dossiers en retard, et ai aussi contribué à l'archivage de dossiers qui avaient été traités par des officiers. Enfin, j'ai occupé le rôle d'officier d'état civil stagiaire lors d'une audition de fiancé·e·s, situation de recherche inconfortable sur laquelle je suis revenue dans deux articles (Lavanchy 2012, 2013a).
} 
tion des régulations du mariage, dans un contexte marqué par divers changements législatifs ${ }^{8}$. Mené en Suisse romande ${ }^{9}$, le travail ethnographique a permis de partager le quotidien professionnel des officiers travaillant dans six offices régionaux, pour des durées respectives allant de deux semaines à deux mois. L'histoire de M. Armand, qui est résumée puis analysée à la suite, résulte d'un entretien effectué dans le cadre d'un projet portant sur les trajectoires d'intégration ${ }^{10}$.

\section{Des fiancé·e·s aux conjoint·e·s}

L'ethnographie de l'état civil a montré l'importance que prennent les impressions des agents sur les couples, impressions échangées dans des moments informels. Ainsi Dominique décrit une cérémonie de mariage particulièrement «belle » à ses yeux en parlant du « petit plus en plus, quand il y a des émotions, quand l'amour, ça coule de leurs yeux, ça se voit » (notes de terrain, 15 mai 2010). Si l'idée qu'amour et mariage vont de pair est un phénomène historiquement situé (Coontz, 2005; d’Aoust, 2013), suivre les officiers au quotidien en révèle la prégnance. Réputé intangible, l'amour doit devenir manifeste pour garantir que les fiancé·e·s ne se marient pas « pour les papiers » (Claude, 27 avril 2010).

Dépister les mariages dits de convenance, ou mariages «blancs », est devenu une obligation légale avec l'introduction d'un nouvel article de loi, en vigueur depuis le $1^{\text {er }}$ janvier 2009. Il prévoit une procédure spécifique à laquelle les officiers recourent en cas de soupçon. Dans les faits, ces mesures administratives sont centrées sur les couples dits " mixtes ", définis par les officiers comme constitués par une personne 《 suisse » et une personne "étrangère " ${ }^{11}$. Sonder ce qu'il y a " dans leurs têtes et dans leurs cœurs » (Claude, 27 avril 2010) devient une tâche professionnelle qui repose sur les « impressions » : « C'est impossible de savoir ce qu'il y a dans les cœurs [mais] on a les impressions... ça se voit en fait, nous, on le voit tout de suite »(Stéphane, 22 mars 2010).

Les impressions des officiers, leur feeling se voient ainsi inclus dans l'activité professionnelle comme outils légitimes. Si les pratiques administratives permettent de créer des conjoint.e.s à partir de deux fiancéee-s, elles instituent (Bourdieu, 1982) surtout une ligne de démarcation entre les couples qui peuvent prétendre se marier et les autres. Ainsi, des couples en sont exclus d'emblée, comme les couples de même sexe, alors que d'autres, qualifiés de « mixtes », se voient soumis à une procédure spécifique particulièrement intrusive et chicanière, dont l'issue est incertaine (Lavanchy, 2013b).

\footnotetext{
${ }^{8}$ Introduction du partenariat enregistré, lutte contre les mariages de convenance et les mariages forcés, interdiction de mariage pour les « sans-papiers ».

${ }^{9}$ Jura, Fribourg, Genève, Neuchâtel, Valais et Vaud. Voir en ligne, consulté le 5 décembre 2019. URL : http://p3.snf.ch/project-131471.

${ }^{10}$ Voir en ligne, consulté le 5 décembre 2019. UR : http://p3.snf.ch/project-147287.

11 Sur la dimension raciale de la « nationalité », voir Lavanchy 2013, 2015 et 2019.
} 


\section{Le lien conjugal comme mécanisme d'intégration}

La nécessité d'encadrer le mariage est une façon d'ordonner le social autour de la frontière entre " eux » et " nous » (Porqueres i Gené, 2000). De fait, se marier génère des devoirs, comme l'assistance financière entre conjoint.e.s, et des droits, notamment face à l'État. Dans un contexte où celui-ci contrôle l'accès au territoire et à la nationalité, la naturalisation devient un enjeu majeur. Le terme de " naturalisation » désigne l'obtention de la nationalité suisse par des personnes étrangères " sur décision des autorités $»^{12}$; elle n'est pas garantie, et peut être rejetée après examen de la demande. Administrativement, la naturalisation est une procédure complexe, nécessitant l'évaluation d'un certain nombre de prérequis et scellée par un acte politique (Lavanchy, Garros, à paraître). Si l'accès à la nationalité est particulièrement restrictif en Suisse (Arlettaz, Argast, Studer, 2013), se marier ${ }^{13}$ avec une personne suisse permet d'échapper aux aspects les plus difficiles de la procédure de naturalisation, en particulier à l'examen administratif de l'intégration, dont la « réussite $»^{14}$ est la pierre d'achoppement de la naturalisation ordinaire.

En cas de demande de naturalisation facilitée, les autorités fédérales ${ }^{15}$ sont responsables d'apprécier si la « communauté conjugale [est] effective et stable » en recourant à une « demande de renseignement » auprès de personnes de référence. Ces dernières sont invitées par courrier à attester d'une part du fait que « les conjoints donnent une image de couple dans leur vie sociale », et que la personne requérante est « intégrée » dans la « communauté suisse » (figure $\mathrm{n}^{\circ} 1$ ).

\footnotetext{
${ }^{12}$ Loi sur la nationalité, Article 2. En ligne, consulté le 26 juin 2019. URL : https://www.admin.ch/ opc/fr/classified-compilation/20092990/index.html.

${ }^{13} \mathrm{Si}$ les partenaires non suisses obtiennent généralement un permis de séjour, il importe de souligner qu'ils et elles ne peuvent demander la naturalisation facilitée, réservée aux conjoint·••s hétérosexuel|le·s. Introduire des dispositions identiques pour les couples de même sexe est l'un des éléments qui figurent dans la proposition limitée de mariage " pour tous », que va prochainement examiner le Parlement (en ligne, consulté le 26 juin 2019. URL : https://www.parlament.ch/ press-releases/Pages/mm-rk-n-2019-02-14.aspx?lang=1036).

${ }^{14}$ Article 12, Loi sur la Nationalité. En ligne, consulté le 26 juin 2019. URL : https://www.admin.ch/ opc/fr/classified-compilation/20092990/index.html.

${ }^{15}$ II s'agit plus précisément du Secrétariat d'état aux migrations (SEM), anciennement Office fédéral des migrations (ODM, cf. facsimilé ci-dessous).
} 


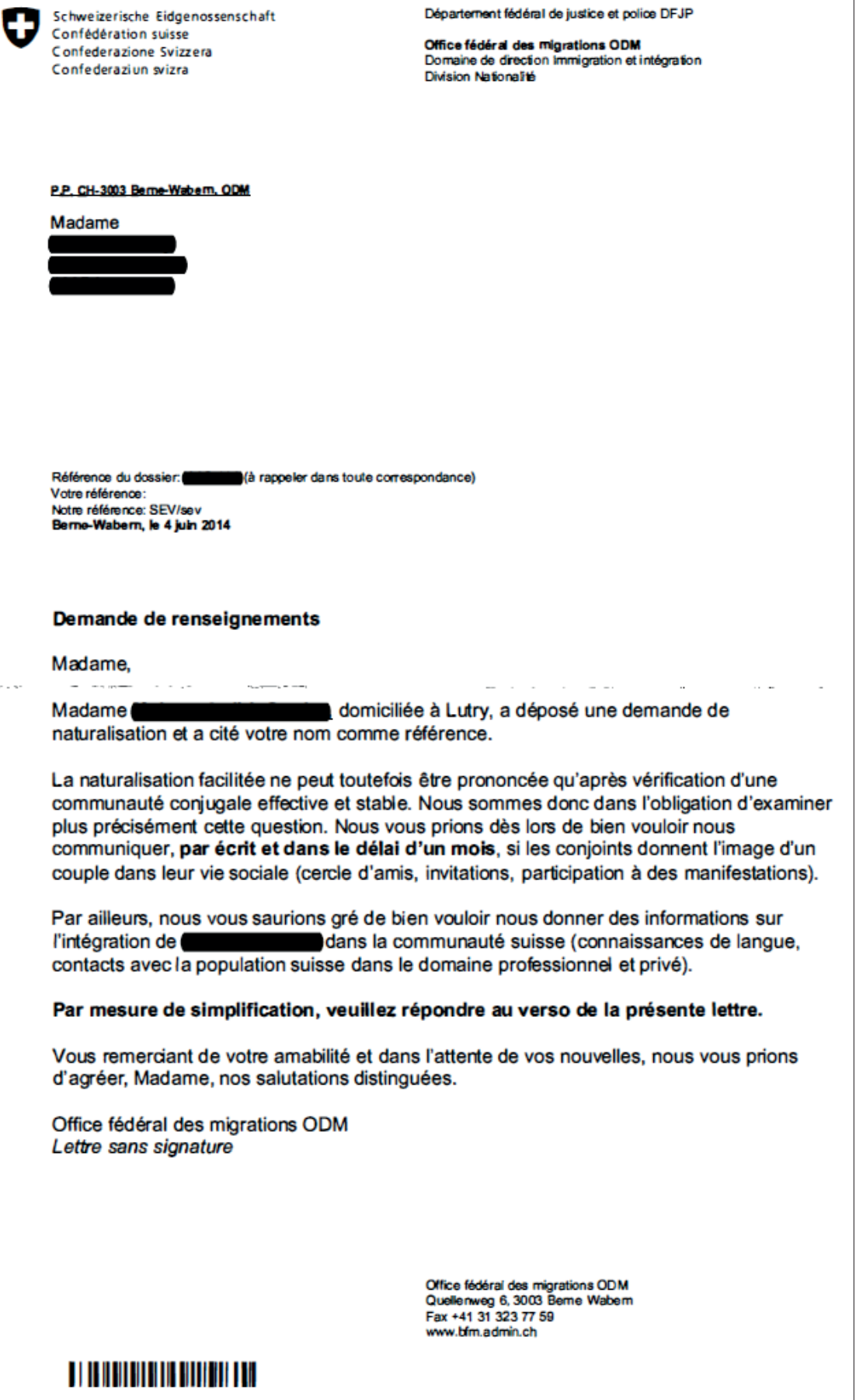

FIGURE N¹ : FACSIMILÉ ANONYMISÉ D'UN COURRIER DE DEMANDE DE RENSEIGNEMENTS DANS LE CADRE D'UNE PROCÉDURE DE NATURALISATION FACILITÉE, 4 JUIN 2014

Ici, l'intégration est laissée à l'appréciation des " personnes de référence » mentionnées par les candidat·e·s à la naturalisation. Les témoins doivent attester que les 
conjoint.e.s donnent « l'image d'un couple». C'est donc le lien conjugal qui, au cours du temps, remplit un rôle d'intégration au corps national.

Si la naturalisation facilitée, qui peut être demandée après cinq années de mariage, fait l'objet d'une procédure qui ne dépend pas de l'état civil, elle plane déjà sur le travail des officiers lors de la procédure de mariage : « Il faut protéger notre pays », « il ne faut pas brader notre pays en acceptant des mariages que pour les papiers », m’ont-ils répété. De leurs propos émerge une catégorie particulière de mariage, celle des « mariages gris ». Cette expression désigne les couples formés par une femme suisse, « amoureuse » et " sincère », qui serait trompée par son fiancé étranger, « froid » et " calculateur », uniquement intéressé à « rester en Suisse » (Lavanchy, 2015). La situation est perçue comme d'autant plus douteuse lorsqu'elle se répète :

Cette femme, elle veut marier un Africain. Mais c'est déjà la deuxième fois. Elle a déjà divorcé d'un Africain avant. Alors, moi, je me demande, non mais je me demande vraiment : pourquoi elle choisit toujours des Africains ? Si elle n'arrive pas à se trouver un mari ici, pourquoi elle ne va pas là-bas ? (Camille, 9 décembre 2009)

La remarque de Camille prend tout son sens lorsque l'on considère qu'avant 1983, les femmes suisses épousant un mari non suisse perdaient automatiquement leur nationalité, et ne pouvaient naturaliser leurs époux, alors que les épouses étrangères d'hommes suisses devenaient automatiquement et immédiatement suisses (Studer, 2004). Notons que jusqu'en 2011, les épouses continuaient de devoir substituer, lors du mariage, leur lieu d'origine par celui de leur mari ${ }^{16}$. L'analyse des pratiques administratives dirigées vers les couples mixtes montre que les effets du lien conjugal ne sont pas symétriques : le lien conjugal semble fonctionner comme un mécanisme qui éloignerait les femmes suisses de « l'ici » du corps national, susceptibles d'appartenir à « là-bas », à l'ailleurs dont provient leur mari, plutôt que d'intégrer leur conjoint. Audelà de la symétrie légale, l'exercice administratif dessine les contours d'une parenté organisée différemment pour les femmes et les hommes.

\section{Après le couple : ce que la séparation révèle du lien conjugal}

Pour mieux comprendre le rôle du lien conjugal dans la production de la famille nationale, il convient de considérer ses effets sur la durée. Le récit de $M$. Armand, retranscrit ci-dessous, en éclaire certains aspects :

Quand je suis venu ici, j'ai demandé l'asile politique. Puis j'ai rencontré une femme, et au bout de quelque temps on s'aimait, on s'est mariés. J'ai retiré [ma demande de] « réfugié politique ». Au bout de cinq ans, on a demandé la naturalisation [facilitée] suisse, ils me l'ont accordée après un an, un an et demi. Je suis

\footnotetext{
${ }^{16}$ Voir le site de l'administration fédérale, en ligne, consulté le 17 juin 2019, URL : https://www.admin.ch/opc/fr/official-compilation/2012/2569.pdf. En Suisse, le lieu d'origine, transmis de manière patrilinéaire, a une signification administrative comparable à celle du lieu de naissance dans la plupart des autres pays.
} 
resté avec ma femme à peu près huit ans, et puis j'ai rencontré une Chinoise. [...] J'étais toujours marié, je ne sais pas comment je vais expliquer, c'était un coup de foudre, j'ai déménagé avec ma copine chinoise. [...] Après quelques mois, ça a posé des problèmes. [Les autorités fédérales à] Berne ont menacé de retirer ma naturalisation [...]. Elles m'ont accusé d'un mariage blanc. Pourtant ça a existé, avec ma femme quand on était chez nous, je ne sais pas, on avait plein plein plein de choses en commun. On est allés au tribunal fédéral [qui a cassé la décision de retrait de nationalité pour vice de forme], ça a duré à peu près cinq ans, cinq ans et demi. À Berne, ils ont dû de refaire leur décision et ils nous ont demandé encore quelques témoins [...], [qui] devaient dire par écrit comment ils nous connaissaient. Berne nous a demandé cinq six témoins, je crois qu'on a à peu près trente personnes qui ont envoyé des lettres. Comme quoi ils ont confirmé que cette famille était ensemble, qu'on vivait ensemble. [...] Avec la Chinoise, au bout d'une année c'était fini. [J'étais de nouveau avec] ma femme, souvent elle disait : «À Berne, ils n'ont jamais trompé leur femme ? Ils ne sont jamais allés à droite à gauche ? On n'a rien fait d'illégal. Mon mari a eu un coup de foudre pour quelqu'un d'autre, et au bout d'une année, il est revenu ! ». [...] C'est grâce à elle que je suis toujours là. (M. Armand, 20 mai 2014)

Ce récit jette un éclairage sur la façon dont une potentielle rupture du lien conjugal menace l'appartenance au corps national. Après une séparation d'avec son épouse suisse, M. Armand se voit confronté à une procédure de retrait de nationalité. En raison de son déménagement, il est soupçonné soit d'avoir fait une déclaration mensongère quant à l'« effectivité » de la communauté conjugale, donc celui d'un mariage « blanc », dans lequel la relation interpersonnelle aurait été purement pécuniaire ; soit d'une déclaration mensongère quant à la « stabilité » de la communauté conjugale, le soupçon étant alors que les personnes étaient sincères au moment du mariage, puis que leur amour se serait éteint, mais qu'elles seraient restées mariées durant cinq ans pour permettre la naturalisation.

Ce récit donne d'autres indications sur ce que recouvrent l'effectivité et la stabilité. Lorsque M. Armand souligne : "Pourtant ça a existé, avec ma femme quand on était chez nous, je ne sais pas, on avait plein plein plein de choses en commun », et qu'il précise que de nombreux témoins ont « envoyé des lettres [pour confirmer] que cette famille était ensemble, qu'on vivait ensemble ", il se réfère à ce que le couple a partagé. Il s'agit ici non seulement des sentiments, mais d'être ensemble physiquement. En creux se dessine ici le rôle des relations sexuelles pour faire couple, et comme mécanisme d'intégration. Légalement, elles ne sont pas nécessaires pour fonder l'union conjugale, comme l'atteste un arrêté du Tribunal fédéral qui précise que "vivre ensemble » consiste à partager « le lit, le toit et la table $»^{17}$, mais n'implique pas de consommer sexuellement le mariage. Elles n'en restent pas moins socialement et administrativement considérées comme attendues et nécessaires pour constituer un vrai couple.

\footnotetext{
${ }_{17}$ Jugement du Tribunal fédéral du 3.12.2010. En ligne, consulté le 5 mai 2019. URL : http://www. servat.unibe.ch/dfr//bger/101203_5A_803-2010.html.
} 


\section{Des substances et de leur circulation}

L'apport fondamental des nouvelles études de la parenté est qu'elles révèlent l'importance de la circulation de « substances » pour constituer les liens de parenté (Carsten, 2013), substances qui peuvent être matérielles, lorsque ce sont des fluides comme le sang ou l'ADN qui circulent entre parents, mais aussi les nourritures ; ou immatérielles, comme le sont des qualités telles que l'esprit, certaines compétences, etc. C'est dans cette perspective que l'on peut comprendre ce que révèle la gestion administrative des couples sur ce qui génère le lien conjugal. La procédure de mariage et celle de la naturalisation indiquent que quelque chose circule dans le lien conjugal : pour permettre à la parenté de déployer des effets sociaux, légaux et administratifs, pour intégrer au corps national des conjoint.e.s non suisses, il faut que circulent entre les conjoint.e.s substances émotionnelles et substances sexuelles.

L'importance accordée conjointement aux impressions, à ce qu'il y a " dans les cœurs » et à " ce qui se voit » indique une caractéristique de l'apparentement par mariage qui se joue à la fois à l'intérieur des corps et dans leur apparence. C'est l'adéquation, telle que perçue par l'officier d'état civil chargé d'évaluer le dossier, entre ce qui est ressenti et ce qui est montré qui fonde le «bon », car véridique, lien conjugal. Les procédures de dépistage des mariages «blancs » accordent une place particulièrement explicite à l'amour. Sa présence devient visible lorsqu'il déborde - lorsqu'il « coule de leurs yeux ». L'amour est ainsi posé en contrepoint de «l'intérêt», qui suppose à la fois un gain pécuniaire et légal, puisqu'il permet de rester sur le territoire national.

Si la circulation des sentiments - comparables à des substances immatérielles échangées entre les fiancé·e·s et qui débordent du dedans vers le dehors - est centrale, c'est aussi parce qu'elle reflète la circulation d'autres fluides entre les corps conjugaux. Comme le montre Maya Mayblin (2013), l'amour joue un rôle tant métaphorique que métonymique par rapport aux substances échangées lors des relations sexuelles. En s'intéressant à l'alliance, à ce que David Schneider (1980) appelle l'ordre de la loi, par opposition à l'ordre de la nature qui apparente les parents à leurs enfants par le biais du «sang », transmis de manière intergénérationnelle, l'analyse montre que le lien conjugal suppose lui aussi la circulation de substances.

Il est particulièrement intéressant de souligner ici que la relation conjugale est porteuse de significations et de conséquences en elle-même. Indépendante de la procréation, elle se déploie dans une logique intra-générationnelle : ce qui permet de créer le lien conjugal, dont découle une possible appartenance nationale, c'est bien ce qui se passe entre les deux conjoint·e.s - ce qui circule d'elle à lui, de lui à elle. Malgré leur proximité, les injonctions à la conjugalité et à la parentalité, à la base du système de pensée hétérosexiste (Tin, 2003) qui prédomine aussi en Suisse, sont distinctes l'une de l'autre. Sous cet angle, il s'avère que la relation conjugale façonne les corps en en modifiant la nature : l'intégration à la communauté nationale par la naturalisation est une véritable incorporation. L'absence de relations sexuelles et/ou d'amour renvoie au mariage « blanc », expression qui rappelle l'absence de traces de relations sexuelles sur des 
draps immaculés. Il est ici révélateur que le manque de relations sexuelles ait comme conséquence le doute sur l'intégration de la personne étrangère, voire même qu'il soit perçu comme un manque effectif d'intégration. En ceci, mon propos entend éclairer de manière novatrice les études sur le continuum entre appartenance familiale et appartenance nationale, en permettant de comprendre que le corps national est façonné par des logiques intra-générationnelle, alors que les analyses se réduisent souvent à sa dimension intergénérationnelle (voir par exemple Roux, Courduriès, 2017).

\section{Conclusion : faire couple par le corps}

L'analyse de la gestion administrative du mariage et ses effets légaux met en lumière la complexité de ce qui lie les conjoint·e·s, et montre comment l'ordre de la loi et celui de la nature sont intimement imbriqués. Généré par un événement particulier, performatif, celui de la cérémonie de mariage, le lien conjugal se développe dans une temporalité plus longue qui est nécessaire pour qu'il déploie tous ses effets.

S’il est réputé intangible, "l'amour » regroupe la dimension, visible, des comportements et des attitudes de l'un envers à l'autre. Ainsi, les témoins sollicités lors des procédures de naturalisation facilitée doivent attester de « l'image » du couple dans sa « vie sociale », par rapport à leur « cercle d'amis, [aux] invitations, [à leur] participation à des manifestations » (figure 1). Cette définition des "bons couples $»^{18}$ souligne que ce qui circule permet une transformation des corps autrefois étrangers l'un à l'autre en corps apparentés. Si les conditions sont réunies, le lien conjugal est suffisamment efficace pour intégrer les conjoint·e·s non suisses à la communauté nationale, en les «naturalisant».

Si elle laisse dans l'ombre ce que le lien conjugal signifie pour les conjoint.e.s et leur entourage, l'anthropologie des pratiques administratives montre que les attentes sociales liées à la bonne conjugalité permettent une discipline des couples autour du genre et de la sexualité. L'attention administrative se concentre sur les mariages " gris » : la circulation se doit d'être symétrique entre les fiancé.e.s. Or les femmes sont décrites comme plus facilement malléables ou influençables - d'une part parce qu'elles sont vues comme potentiellement naïves et crédules ; d'autre part en raison de leur nature même, qui les rendrait plus susceptibles de changer de nature au cours du corps à corps avec des époux étrangers, conférant à leur appartenance nationale une volatilité problématique.

\section{Bibliographie}

Aeby G., Berthod M. (2011), « Entrer et sortir des institutions », Tsantsa, vol. 16, p. 6-17.

Arlettaz G., Argast R., Studer B. (2013), Le droit d'être suisse. Acquisition, perte et retrait de la nationalité de 1848 à nos jours, Lausanne, Antipodes.

\footnotetext{
18 L'article ne considère pas ici la question de la classe sociale, qui est pourtant importante, et ce d'autant plus que cette forme d'homogamie connaît une forte augmentation (Ravazzini et al., 2019).
} 
Boillet, V., De Luze, E. (2018). « Les effets de la gestation pour autrui à caractère international en Suisse : analyse de la jurisprudence du Tribunal fédéral », in V. BOILLET, M. RocA I EsCODA, E. De LuZE (dir.) La gestation pour autrui : approches juridiques internationales, Limal, Bâle, Anthemis, Helbing Lichtenhahn Verlag, p. 143-181.

BOURDIEU P. (1982), « Les rites comme actes d'institution », Actes de la recherche en sciences sociales, vol. 43, p. 58-63.

Carsten J. (2011), «Substance and Relationality: Blood in Contexts », Annual Review of Anthropology, vol. 40, p. 19-35.

CARSTEN J. (2013), «Introduction: Blood Will Out», Journal of the Royal Anthropological Institute, vol. $19, \mathrm{n}^{\circ} \mathrm{S} 1, \mathrm{p}$. S1-S23.

Coontz S. (2005), Marriage, a History: From Obedience to Intimacy or how Love Conquered Marriage, New York, Viking.

D'Aoust A.-M. (2013), « In the Name of Love: Marriage Migration, Governmentality, and Technologies of Love », International Political Sociology, vol. 7, p. 258-274.

FASSIN E. (2008), «États-Unis/France. L'amour du même et l'amour de l'autre. Le “mariage homosexuel" entre famille nationale et question raciale », in V. Descoutures (dir.) Mariages et Homosexualités dans le monde, Paris, Éditions Autrement, p. 99-111.

Hertz E., Martin H., Valli M. (2004), " Le “feeling” : une logique sous-jacente au fonctionnement de l’État providence », Aspects de la sécurité sociale, vol. 3, p. 12-21.

Hill Collins P. (2001), « Like One of the Family: Race, Ethnicity, and the Paradox of US National Identity », Ethnic and Racial Studies, vol. 24, p. 3-28.

LAURENS S. (2008), « Les agents de l'État face à leur propre pouvoir. Éléments pour une microanalyse des mots griffonnés en marge des décisions officielles », Genèses, vol. 3, p. 26-41.

Lavanchy, A. (2012) " Going under Cover? Ethics, Transparency and Witnessing in Researching Institutions ", GRED, Ethical case discussions in the ethnological research. En ligne, consulté le 12 décembre 2019. URL : http://www.seg-sse.ch/ pdf/2012-10-30_Lavanchy.pdf.

LAVANCHY A. (2013a), « Dissonant alignments. The ethics and politics of researching state institutions», Current Sociology, vol. 61, nº 5-6, p. 677-692.

LAVANCHY A. (2013b), « L'amour aux services de l'état civil : régulations institutionnelles de l'intimité et fabrique de la ressemblance nationale en Suisse », Migration sociétés, vol. 25, p. 61-94.

Lavanchy A. (2014), "Die Gefühlswelt des Gesetzes: die kritische Umsetzung von eherechtlichen Vorschriften im Zivilstandsamt ", FAMPRA - die Praxis des Familienrechts, vol. 15, n 1, p. 92-117. 